\title{
Apropriações psicológicas do campo da saúde do trabalhador: Uma revisão sistemática
}

Cássio Adriano Braz de Aquino. Universidade Federal do Ceará

Mariana Aguiar Alcântara de Brito. Universidade de Fortaleza

Marselle Fernandes Fontenelle. Universidade de Fortaleza

Maxmíria Holanda Batista. Universidade de Fortaleza

Pamella Beserra de Melo. Universidade Federal do Ceará

Samara Silva Silveira. Companhia de Água e Esgoto do Estado do Ceará

\section{Resumo}

O texto investiga formas de apropriação do campo da Saúde do Trabalhador pela Psicologia através de uma revisão sistemática de 37 artigos publicados no Brasil no período de 2010 a 2015, disponíveis no Portal de Periódicos da Capes e no Portal de Periódicos de Psicologia (PePSIC). Utilizaram-se como descritores "Saúde do Trabalhador" e "Psicologia". Observou-se estabilidade das produções nesse campo, com destaque à utilização de relato de pesquisas, uso de métodos qualitativos e base teórica fundamentada na perspectiva dos estudos e pesquisas em subjetividade e trabalho. As vias de apropriação da categoria trabalho pela Psicologia concentram-se nas vertentes Social e Clínica, com predomínio das publicações nas regiões Sudeste e Sul e foco em pesquisas com trabalhadores da saúde. As formas de apreensão da Psicologia no campo da Saúde do Trabalhador são diversificadas, demandando atenção com a interdisciplinaridade, apontando para a ampliação do diálogo entre saúde mental e saúde do trabalhador.

Palavras-chave: saúde do trabalhador; psicologia; trabalho; produção científica.

\begin{abstract}
Psychological appropriation of occupational health field: a systematic review. The appropriation routes of Occupational Health field by Psychology are investigated through a systematic review of 37 national papers in the period of 2010-2015, available in the Capes journals portal and in PePSIC database. "Occupational Health" and "Psychology" were used as descriptors. Production stability in the field of Occupational Health was observed, with emphasis on the use of research reports by means of qualitative methods and theoretical basis based on the perspective of studies and research on subjectivity and work. Regarding the types of appropriation of the category work by Psychology, it was possible to verify a concentration in social and clinical aspects with predominance of publications in the southeastern and southern regions of Brazil, focusing on research with health workers. The forms of appropriation of Psychology in the Occupational Health field are quite diverse, demanding a caution with interdisciplinarity and pointing to the expansion of the dialogue between mental health and occupational health.
\end{abstract}

Keywords: occupational health; psychology; work; scientific production.

\section{Resumen}

Apropiación psicológica del campo de la salud de los trabajadores: una revisión sistemática. El texto investiga la apropiación del campo de la Salud del Trabajador por la Psicología a través de una revisión sistemática de 37 artículos publicados en Brasil entre 2010-2015 en el portal de Capes y en el base datos PEPSIC. Los descriptores utilizados fueran "Salud de los Trabajadores" y "Psicología". Se observó una estabilidad de las producciones en ese campo, con destaque en los de informes de investigaciones, por medio de métodos cualitativos enfatizando los estudios e investigaciones sobre la subjetividad y el trabajo. Las formas de apropiación del trabajo por la Psicología fueron principalmente la Social y la Clínica, con fuerte incidencia de publicaciones en las regiones sudeste y sur de Brasil, privilegiando investigaciones con trabajadores de la salud. Las formas como la Psicología tiene repercutido en el campo de la salud son muy diversas, exigiendo una atención con foco interdisciplinario, señalando hacía la expansión del diálogo entre la salud mental y la salud ocupacional.

Palabras clave: salud del trabajador; psicología; trabajo; producción científica. 
As transformações no mundo do trabalho têm sido diversas, abarcando as relações sociais, as tecnologias do processo de produção e a gestão da organização do trabalho. Todas essas mudanças relacionam-se a diferentes contextos políticos, econômicos e socioculturais. Contudo, os processos de dominação e desgaste desvelado pelo trabalho permanecem, mesmo que por vezes metamorfoseados (Seligmann-Silva, 2011).

O trabalho vem sendo considerado tanto um fator constitutivo na formação da doença quanto desencadeante ou contributivo do adoecimento. Exerce ainda função psicológica por excelência no processo de humanização do trabalhador e funciona como operador de saúde através da criação de novas formas de viver (Clot, 2007; Seligmann-Silva, 2011).

Elegemos aqui, como matriz de compreensão da relação saúde-trabalho, o campo da Saúde do Trabalhador, abordagem oriunda da Medicina Social Latino-Americana e da Saúde Coletiva da década de 1970 que destacam os determinantes sociais na relação saúde-doença dos trabalhadores e privilegiam o processo de trabalho como categoria de análise. Essa área busca conhecer e intervir, de forma interdisciplinar e multiprofissional, nas relações de trabalho e nos processos de saúde e doença, situando os trabalhadores como sujeitos ativos, agentes de mudanças, capazes de compartilhar as vivências de trabalho no coletivo e participar das transformações necessárias para a promoção da saúde em seus contextos laborais (Lacaz, 2007, 2016; Sato, Lacaz, \& Bernardo, 2006).

Nas últimas décadas, esse campo buscou implementar várias ações e práticas no sentido de fortalecer as políticas públicas de atenção integral em saúde do trabalhador. No entanto, os avanços não foram capazes de superar os entraves interinstitucionais inerentes à consolidação, no Sistema Único de Saúde (SUS), das ações na área. Citam-se como algumas dessas barreiras a desarticulação dos programas executados pelos Ministérios da Saúde, do Trabalho e da Previdência Social e a fragilidade do controle social representado pelos movimentos sindicais e pela sociedade civil organizada (D. Costa, Lacaz, Jackson Filho, \& Vilela, 2013; Lacaz, 2007, 2016).

No que diz respeito à saúde mental, a Organização Mundial da Saúde (OMS) estima índices de 30\% de transtornos mentais na população trabalhadora ocupada até 2020, com prevalência para o aumento nos casos de depressão, tornando-se esta a segunda maior causa de afastamento por incapacidade, perdendo apenas para as doenças isquêmicas do coração (Jardim, Ramos, \& Glina, 2010).

No contexto de crise econômica mundial, ampliada no Brasil pela crise política vigente, observa-se o avanço nos índices de desemprego, a intensificação da precarização do trabalho e o agravamento das desigualdades sociais (Chaves, 2016). Todo esse cenário acaba servindo como justificativa para desviar a atenção e o cuidado com a saúde em nome da sobrevivência, mesmo que precária, no trabalho.

A saúde do trabalhador no Brasil está sendo vulnerabilizada ante o crescente processo de precarização laboral e a ameaça da perda de direitos que sequer foram alcançados em sua plenitude. A ampliação das novas formas de contratação estabelecidas pela lei $n$. 13.429, sancionada parcialmente em março de 2017 pelo então presidente Michel Temer, tornando a terceirização irrestrita, a redefinição do conceito de trabalho análogo à escravidão, a disseminação da remuneração flexível vinculada a metas de produção, a flexibilização da jornada de trabalho, ao prever que o trabalhador reduza "seu tempo de vida a tempo de trabalho" (Alves, 2016, p. 46) são exemplos de retrocessos em alguns dos direitos trabalhistas, cujo efeito social estará na ampliação dos adoecimentos laborais (Aquino, Saboia, Melo, Carvalho, \& Ximenes, 2016).

No modelo toyotista, que mobiliza a difusão do trabalho imaterial e passa a atuar com predomínio no setor de serviços, ampliam-se os danos psicossociais para o trabalhador que, em um cenário de constante ameaça de desemprego, sente-se convocado a desenvolver sua polivalência, agilidade e flexibilidade (Navarro \& Padilha, 2007, p. 19).

Desse modo, o desgaste do trabalhador se dá pelas exigências extremas de seus aspectos cognitivos e afetivos a serviço do trabalho, dentro e fora dele. "A exploração no mundo do trabalho toma outros contornos, apresentando novas exigências quanto ao uso de capacidades criativas e adaptativas - características subjetivas - da força de trabalho" (Osório da Silva, 2007, p. 2). As novas sutilezas do sistema de gestão transbordam para a vida cotidiana, causando ansiedade e sofrimento psíquico (Seligmann-Silva, 2011).

A contribuição da Psicologia para o campo da Saúde do Trabalhador está centrada especialmente nas bases da Psicologia Social e da Saúde, da Psicologia Social do Trabalho e da Psicologia Clínica, com ênfase na Psicanálise. Cada uma dessas bases produz contribuições específicas e múltiplas e reflete a diversidade 
característica da(s) Psicologia(s) enquanto ciência (Centro de Referência Técnica em Psicologia e Políticas Públicas [CREPOP], 2008).

A partir da ampliação do campo de atuação da Psicologia, notadamente na relação trabalho e saúde mental, algumas contribuições teóricas ganham destaque, como as propostas por Jacques (2003), Borges, Guimarães e Silva (2013) e Bendassoli (2011), que serviram de fio condutor da análise aqui empreendida.

Diante de toda essa abrangência de estudos e instigado pela proposta de Bendassolli (2011) em discutir criticamente as formas de apropriação psicológica do trabalho que sugere, ainda, uma visão de saúde ampliada, rompendo com uma possível linearidade e com a restrição da saúde à perspectiva clínica, este artigo se propõe a investigar quais as formas de apropriação do campo da Saúde do Trabalhador dentro das abordagens teórico-metodológicas da Psicologia, explorando uma revisão sistemática de artigos científicos nacionais produzidos entre 2010 e 2015.

A escolha de artigos nacionais se justifica pelo interesse em conhecer a contribuição da Psicologia no Brasil, especialmente diante do cenário de crescente flexibilização e de indícios de uma despolitização do campo da Saúde do Trabalhador, gerada pela crise de representação política, social e sindical que se abateu sobre a sociedade brasileira nos últimos anos (Lacaz, 2016).

\section{Método}

O percurso metodológico utilizado neste artigo foi a revisão sistemática. "A revisão sistemática é um método que permite maximizar o potencial de uma busca, encontrando o maior número possível de resultados de uma maneira organizada" (Costa \& Zoltowski, 2014, p. 56). É um tipo de investigação focada em uma questão bem delimitada, que, por possibilitar o acesso a estudos sistematizados através de dados secundários, pode colaborar em processos decisórios acerca de eventos clínicos e em delineamentos de futuras pesquisas, sendo ainda classificada como contribuição original em grande parte das revistas científicas (Pereira \& Galvão, 2014). Optamos por essa estratégia de pesquisa por identificar também que a difusão desse expediente é ainda preambular, ao menos com esse escopo, nas produções nacionais.

Demarcada a questão a ser pesquisada a partir da investigação das abordagens teórico-metodológicas da Psicologia, escolhemos publicações em formato de artigos científicos disponíveis no Portal de Periódicos Capes, uma biblioteca virtual que permite o acesso completo dos textos e reúne as principais bases referenciais da produção nacional, e no Portal de Periódicos Eletrônicos de Psicologia (PePSIC) , uma biblioteca virtual em saúde e um portal específico para a ciência psicológica, o qual tem como objetivo ampliar a visibilidade de produções geradas nos países da América Latina (Hohendorff, DeSousa, Pereira, \& Koller, 2016). Para a realização das buscas, elegemos o descritor "Saúde do Trabalhador" a partir da consulta à Biblioteca Virtual em Saúde (BVS-Psi).

Na pesquisa realizada em abril de 2016 ao Portal de Periódicos Capes e ao portal PePSIC, encontramos 2.971 resultados na literatura nacional sobre o assunto. Para refinar a procura de forma coerente com a problemática aqui retratada, foram utilizados, na busca final, os termos "Saúde do Trabalhador' \& "Psicologia", considerando apenas descritores em português pelo interesse exclusivo em publicações de abrangência nacional. Com o acréscimo desse ordenador booleano, reduziu-se a pesquisa para 138 resultados. Devido à celeridade das mudanças no cenário político, somadas ao impacto da crise econômica que teve seu auge entre 2008 e 2009, com repercussões a partir de 2010, circunscrevemos a investigação entre os anos 2010 e 2015 a fim de considerar as publicações mais recentes, como forma de averiguar a atualização das informações do campo teórico na área da Psicologia. Dessa forma, ao incluir a delimitação temporal de busca de textos publicados nesse período, alcançou-se um resultado de 50 artigos nas fontes já referidas. Treze desses estudos, no entanto, foram excluídos porque se apresentavam de forma duplicada nas bases acessadas. Os 37 artigos encontrados na pesquisa final foram analisados a partir das seguintes informações: título, ano de publicação, revista, quantidade de autores, região de origem do autor principal, área de formação do autor principal, palavras-chave, sujeitos da pesquisa, vias de apropriação da Psicologia (Organizacional, Social, Clínica ou outros), pressupostos teórico-metodológicos (tipo de tratamento de dados, base teórica e tipo de pesquisa) para subsidiar a discussão dos resultados.

\section{Resultados e discussões}

Com base nos resultados referentes ao ano de publicação, constatamos pouca variação no número de artigos publicados - exceção feita a 2013, quando se 
verifica o menor número de publicações no período em estudo. Verificamos que a temática acerca da apropriação da Psicologia no campo da Saúde do Trabalhador tem se mantido estável, dado que a Psicologia é uma área com foco na saúde e no bem-estar (Gondim, Borges-Andrade, \& Bastos, 2010; Tonetto, 2008).

Na figura 1, referente à distribuição dos artigos por região na qual o autor principal possui vínculo institucional, há uma predominância $(54,5 \%)$ desse vínculo com instituições localizadas na região Sudeste. Esse dado aponta para uma tendência à concentração de pesquisadores, o que pode levar a uma análise enviesada e parcial acerca do tema da apropriação da Psicologia do campo da Saúde do Trabalhador, pois aspectos relativos à diversidade das realidades socioculturais, à heterogeneidade dos contextos de trabalho e às condições específicas de saúde dos trabalhadores tendem a não ser contemplados.

É importante notar que, seguindo essa linha, há uma forte concentração dos programas de pós-graduação naquela região, com a presença de 1.952 deles, o que corresponde a $44,5 \%$ do total no Brasil (Capes, 2017). A pós-graduação em Psicologia reflete, de um modo geral, a realidade brasileira. Os dados do relatório da avaliação da Capes (2013) ressaltam uma desigualdade regional, situando os Estados do Sudeste como detentores de 50,7\% desses programas. Além disso, o relatório destaca que três subáreas congregam 42,6\% das linhas de pesquisa, a saber: Psicologia Social, Psicologia Clínica e Processos Psicológicos Básicos.

Desse modo, o resultado referente à distribuição de artigos por região sugere que a concentração dos programas de pós-graduação em Psicologia no Sudeste pode favorecer uma maior incidência ali de pesquisas e publicações.

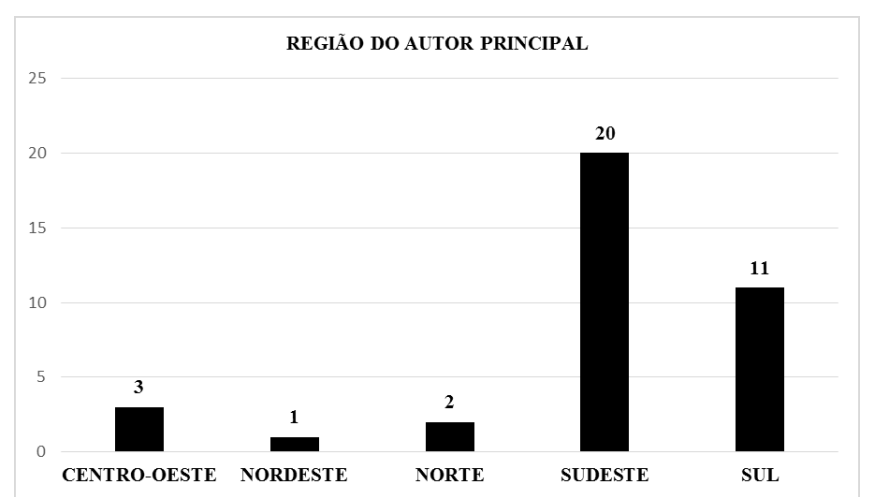

Figura 1. Distribuição dos Artigos por Região do Autor Principal.
As publicações, pelo tipo de tratamento que os dados receberam, foram classificadas em quantitativas, qualitativas ou quanti-qualitativas. Observamos que a ampla maioria dos artigos (70,3\%) utilizou uma análise qualitativa, indicando a ênfase da análise a partir das experiências reais dos sujeitos da pesquisa, bem como na perspectiva interpretativa dos dados por parte do pesquisador (Creswell, 2007). Esse fenômeno evidencia a escolha pela abordagem qualitativa como um caminho tradicionalmente percorrido pelas investigações na área da Psicologia e, em especial, no campo da Psicologia que estuda o trabalho.

Para Borges-Andrade e Zanelli (2014), quem utiliza abordagens qualitativas atribui grande importância ao envolvimento do pesquisador nas organizações de trabalho e no uso dos sentidos humanos como primordial para a interpretação de fenômenos comportamentais, em especial as falas e os discursos presentes nesses ambientes.

A Figura 2 descreve a distribuição dos artigos por modo de apropriação do trabalho ao nível conceitual, elemento fundamental em nossa discussão. Para essa análise, utilizamos como referência Bendassolli (2011). O autor afirma que a via organizacional abrange os estudos relacionados ao desempenho pessoal, grupal e organizacional no trabalho e tem forte vinculação ao contexto no qual o "indivíduo aprende e adquire os meios para se desempenhar eficientemente como profissional" ( $p$. 79). Por sua vez, a via social compreende estudos que abordam o trabalho como fato social, objeto de construção do cotidiano e recurso para a individuação, para a construção do significado de si mesmo e do outro. Nessa via, o trabalho é considerado como atividade que não se reduz ao emprego, e a preocupação dos estudos é na relação entre vida psíquica e trabalho e na centralidade do trabalho. Já a via clínica parte de uma concepção de clínica aplicada ao contexto social, que visa à emancipação dos sujeitos e à transformação dos contextos de trabalho como resposta aos problemas vividos pelo trabalhador em sua experiência laboral.

Identificamos uma concentração das publicações nas vias social e clínica. Isso indica que os estudos, de um modo geral, se preocupam em compreender os aspectos do trabalho e suas influências na constituição da identidade dos sujeitos, assim como buscam transformar as condições objetivas do trabalho por meio do poder de agir dos trabalhadores. Uma parte das pesquisas não foi classificada a partir das vias apresentadas por Bendassolli (2011) porque se detiveram em 
aspectos que guardavam pouca relação com os aportes teóricos utilizados como base da revisão sistemática aqui utilizada.

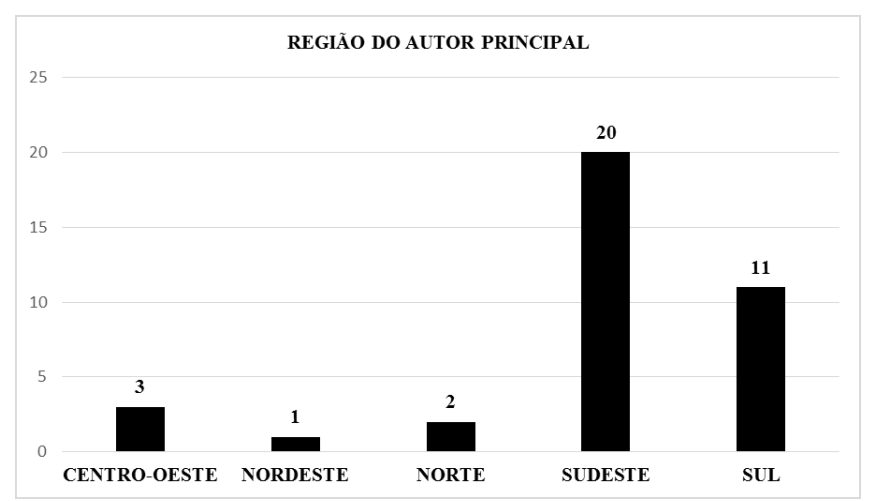

Figura 2. Distribuição dos Artigos por Vias de Apropriação da Categoria Trabalho.

As pesquisas empíricas foram o principal percurso metodológico adotado para se apropriar e se compreender o campo da Saúde do Trabalhador. Notamos que $48,6 \%$ dos artigos optaram por desenvolver essa perspectiva. Incluímos nessa categoria todos aqueles estudos em que os autores descreveram um método pautado no uso de qualquer ferramenta que facilitasse a extração de informações dos sujeitos ou do contexto de trabalho, sejam elas de cunho qualitativo ou quantitativo.

Percebemos que, apesar dos esforços aplicados em realizar pesquisas institucionais que possam vir a subsidiar políticas voltadas para a saúde do trabalhador, revisões sistemáticas e de artigos teóricos permanecem com um quantitativo relevante, representando $32,4 \%$ do total. Esses dados nos levam a refletir que, com as frequentes transformações no mundo do trabalho, faz-se necessário atualizar o campo da Saúde do Trabalhador.

Como sugerem A. B. Costa e Zoltowski (2014), as revisões sistemáticas contribuem para a ampliação dos estudos na medida em que oferecem subsídios para o preenchimento de lacunas ainda existentes. No caso do campo da Saúde do Trabalhador, ajuda os pesquisadores a trilhar diferentes caminhos, desviando de áreas já saturadas e colaborando com novas propostas.

No que se refere à ocupação dos sujeitos pesquisados nos artigos que compuseram essa revisão sistemática, observamos que, de modo coerente com o explicitado na discussão acima, mostra-se relevante a categoria "não se aplica" (37,8\%), que se traduz em pesquisas que não tiveram como foco procedimentos metodológicos com humanos, ou seja, concentraram-se em revisão de literatura ou sistemas eminentemente teóricos. No entanto, apontamos que o segundo grupo de maior interesse entre os pesquisadores está inserido na área da saúde (24,3\%), destoando significativamente em termos quantitativos das outras categorias profissionais estudadas.

Consideramos que a justificativa para tal resultado esteja evidenciada no fato de que os profissionais envolvidos no cuidado com os outros possuem, de modo geral, uma rotina caracterizada por pressões ambientais: um alto grau de cobrança vindo dos superiores, da família dos pacientes e de si mesmo, horários de trabalho indefinidos, infraestrutura precária, insuficiência de suprimentos, demanda excessiva, além da responsabilidade em lidar com o sofrimento do outro e o compromisso de tentar amenizá-lo. No Brasil, esses dados vêm sendo confirmados por meio do desenvolvimento de pesquisas em diversas regiões, nas quais se tem demonstrado que a saúde, a qualidade de vida e o bem-estar de trabalhadores da área da saúde têm sido prejudicados (Leal, Bandeira, \& Azevedo, 2012; Oliveira, Viagnó, Lunardelli, Canêo, \& Juniors, 2010; Roque, Veloso, Silva, \& Costa, 2015; Rosado, Russo, \& Maia, 2015; Silva et al., 2015). Esses autores encontraram relações entre a sobrecarga de trabalho, os indicadores de satisfação e o comprometimento da saúde mental dos trabalhadores de saúde, o que indica a importância e o crescente interesse dos pesquisadores em estudar profissionais dessa área. Em 2009, por exemplo, Moreira, Magnago, Sakae e Magajewski já apontavam para a alta incidência de estresse e síndrome de burnout em indivíduos cujo trabalho estava associado ao contato com pessoas e ao cuidado com outrem, principalmente em profissionais da saúde e da educação.

Na Figura 3, apontamos a distribuição dos artigos por base teórica, à luz da classificação proposta por Jacques (2003) e Borges et al. (2013) sobre os estudos da relação saúde-doença mental e trabalho. Verificamos que a maioria $(48,6 \%)$ dos artigos foi categorizada na perspectiva Estudos e Pesquisas em Subjetividade e Trabalho. A concentração evidencia o foco das publicações nas vivências, no cotidiano, no modo de vida dos trabalhadores, tendo como eixo principal a discussão da relação do homem com o mundo do trabalho. Esses estudos valorizam os aspectos qualitativos das experiências dos indivíduos associados ao processo de saúde-adoecimento e trabalho, bem como privilegiam métodos qualitativos de pesquisa e intervenção. 
Tal característica está em consonância com a discussão sobre o tratamento dos dados, que indica a prevalência do método qualitativo como pressuposto metodológico empregado pela maioria dos artigos analisados nesta revisão. Destacamos também que a Psicodinâmica do Trabalho foi utilizada em $21,6 \%$ dos artigos, representando a segunda perspectiva teórica mais adotada no universo das publicações. Essa abordagem influenciou sobremaneira as pesquisas brasileiras acerca da discussão em saúde e trabalho; ao longo do tempo, incorporou novos saberes na tentativa de dar conta da complexidade dos contextos laborais e direcionou o seu foco aos processos de subjetivação e à análise das estratégias de defesa usadas pelos trabalhadores para lidar com a organização do trabalho profissional.

O interesse por essa perspectiva teórica coaduna-se também com a concentração da perspectiva metodológica qualitativa, pois a Psicodinâmica utiliza como método a escuta, por meio de entrevistas coletivas, e a interpretação, com posterior devolutiva. Verificamos ainda que a recorrência das fundamentações teóricas supramencionadas está em conformidade com os dados descritos na Figura 2, indicando a concentração dos artigos nas vias social e clínica, propostas por Bendassolli (2011).

Contudo, as poucas publicações na perspectiva epidemiológica $(16,2 \%)$ enfraquecem a possibilidade de a Psicologia contribuir com o estabelecimento do nexo epidemiológico e assim subsidiar a luta dos trabalhadores em fazer seus direitos respeitados.

Sarriera et al. (2003), ao realizar estudos com psicólogos pesquisadores de Brasil, Espanha e Argentina sobre suas concepções a respeito da saúde e dos estudos epidemiológicos, apontaram que alguns participantes partem de uma descrição superficial da epidemiologia, muitas vezes vinculada à concepção médica. Os autores alertam para a necessidade de esses profissionais se apoderarem do aporte teórico da epidemiologia e discutirem a aplicação desses estudos especificadamente para sua área, realizando análises críticas a respeito.

Para Bendasolli e Gondim (2014) talvez a forte influência que as diversas correntes qualitativas têm exercido na Psicologia Social do Trabalho favoreceram a ênfase nas investigações da experiência subjetiva dos trabalhadores, isto é, discute-se o sentido do trabalho à luz da história de vida dos sujeitos. Contudo, para os autores, há em alguns casos uma "reificação da narrativa pessoal descolando-a do contexto" (p. 137).
Esse aspecto poderá estar relacionado aos resultados aqui apresentados, que demostram uma predominância das pesquisas em tentar compreender a subjetividade do trabalhador e seu processo saúde-adoecimento. De uma forma geral, os objetivos contemplados pela epidemiologia são o estudo da distribuição dos fatores de riscos (determinantes dos eventos relacionados com a saúde), a descrição das condições de saúde e doença, o mapeamento da prevalência das patologias em determinados contextos e a avaliação do impacto das ações para alterar a situação de saúde (Seligmann-Silva, 2011). Estabelecer o nexo causal saúde-doença mental no trabalho continua sendo uma importante demanda da Psicologia no campo da Saúde do Trabalhador, pois a exigência legal de demonstração do nexo causal entre a patologia e as condições e organização do trabalho é pressuposto necessário para o acesso dos trabalhadores aos benefícios previdenciários (Borsoi, 2007; Jacques, 2003).

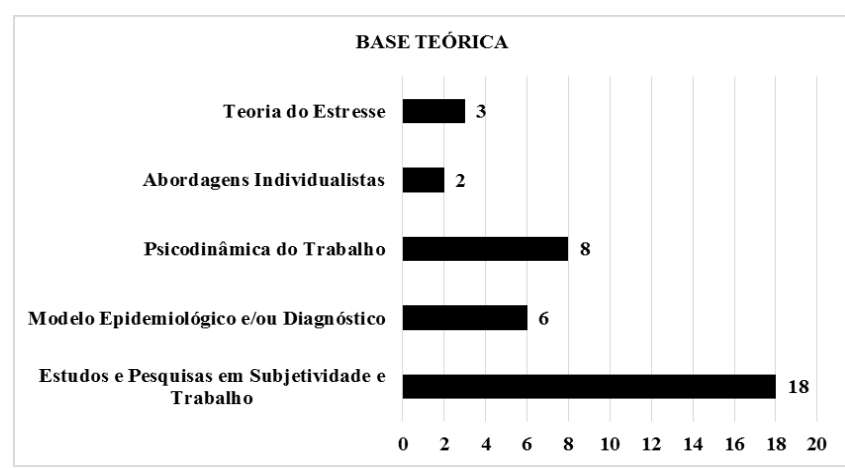

Figura 3. Distribuição dos Artigos por Base Teórica.

Outro resultado que destacamos se refere à prevalência de pesquisas voltadas para a saúde mental com destaque ao contexto do trabalho, totalizando 19 artigos $(51,4 \%)$ com essa aderência.

De acordo com Lima (2016), a procura por serviços em Saúde Mental ocorre prioritariamente por meio dos serviços de urgência, e seu foco está nos impedimentos do indivíduo em relação à vida em geral. Já na Saúde do Trabalhador, os casos são processuais e, muitas vezes, vinculados às características do ofício de uma determinada categoria profissional. No entanto, a autora adverte que, antes da configuração do adoecimento, o trabalhador, com frequência, é encaminhado para a atenção primária ou para o médico do trabalho, recebendo um diagnóstico que geralmente não correlaciona a sua doença com a situação laboral. Esse fenômeno corrobora para dificultar o estabelecimento do nexo causal entre saúde-doença mental e trabalho. 
Além disso, muitos casos de abuso de álcool e outras drogas, além de tentativas de suicídio, aparecem relacionados à demanda da saúde mental, mas são conduzidos sem associação com o contexto laboral (Lima, 2016). Esses dados apontam para a necessidade de maior aproximação entre os estudos da saúde mental e da saúde do trabalhador na Psicologia.

\section{Conclusões}

A presente revisão sistemática apontou para uma estabilização das publicações no campo da Saúde do Trabalhador no período pesquisado, com relativo aumento nos últimos dois anos. Acreditamos que esse aumento de produção está relacionado às medidas provisórias 664 (2014) e 665 (2014), que alteram os critérios de concessão de benefícios previdenciários, bem como à lei 13.429, de março de 2017, que dispõe sobre as regras de contratação de serviços de terceiros. Esses dispositivos legais representaram, segundo Antunes e Praun (2015), a quebra dos direitos dos trabalhadores, a partir da redução dos custos para os empresários, e a desestabilização da classe trabalhadora. Tal fenômeno pode ter influenciado o interesse dos pesquisadores e dos periódicos para o debate sobre as condições de saúde da população trabalhadora nesse contexto caracterizado por fortes mudanças.

Destacamos que as investigações, em sua maioria, caracterizam-se por relatos de pesquisa, com foco nos trabalhadores da área da saúde. Houve uma predominância no emprego de métodos qualitativos e de base teórica alicerçada notadamente na perspectiva de estudos e pesquisas em Subjetividade e Trabalho, seguido pela Psicodinâmica do Trabalho, o que é compatível com o resultado encontrado em relação às vias de apropriação da categoria trabalho pela psicologia, que apresentou concentração nas vertentes Social e Clínica.

A prevalência da distribuição dos artigos nas regiões Sudeste e Sul é marcante, apontando ainda para a escassez de estudos em outras regiões, em especial no Nordeste, embora esta não destoe, em número de programas de pós-graduação, significativamente da região Sul. Dessa compreensão inferimos que a vasta realidade brasileira não é devidamente contemplada pelas discussões acadêmicas atuais.

De forma mais ampla, acreditamos que os modos de inclusão da Psicologia no campo da Saúde do Trabalhador são bastante diversificados e, por vezes, articulados a outros saberes e práticas da saúde, sinalizando para o cuidado constante em agir de forma interdisciplinar sem perder de vista as especificidades de cada vertente.

A ampliação do diálogo entre as áreas da Saúde Mental e da Saúde do Trabalhador aparece como um caminho possível para o fortalecimento da contribuição da Psicologia. Os avanços nos estudos acerca das psicopatologias laborais e das possibilidades de desenvolvimento da saúde - apontados pela perspectiva das clínicas do trabalho - junto com as práticas propositivas de escuta e inclusão - recomendadas pelas clínicas psicológicas - aparecem como vertentes que, se articuladas, podem favorecer a uma inserção profícua da Psicologia na Saúde do Trabalhador. Somados a esses aspectos, o cuidado com o diagnóstico e a construção de pareceres que visibilizem os aportes teórico-metodológicos constantes no nexo causal deve dar sentido à contribuição do saber psicológico.

Sugerimos um exercício perene de aprofundamento acerca das bases epistemológicas de cada saber e fazer psicológico na área; alie-se a isso o compartiIhamento entre os pares de diversas posições teórico-metodológicas, possibilidades de atuação e limites de procedimentos. Promove-se assim um fortalecimento político e ético da categoria do psicólogo no âmbito da saúde, abrindo um espaço aqui também para a consolidação e a valorização da Psicologia.

O reconhecimento de diferenças e similaridades das diversas formas de apropriação psicológica do campo da Saúde do Trabalhador alinhado com o propósito de inclusão dos saberes e das vivências dos sujeitos acerca do desgaste e da saúde relacionados ao trabalho pode contribuir para uma compreensão e uma intervenção no campo de forma mais comprometida com as transformações sociais e com a redução das vulnerabilidades e das injustiças a que estão submetidos os trabalhadores. Diante dos contextos de trabalho adoecedores, pautados nos preceitos neoliberais de flexibilização, racionalização e precarização do mundo do trabalho, faz-se mister conhecer os possíveis posicionamentos da Psicologia na relação saúde e trabalho.

\section{Referências}

Alves, G. (2016). Terceirização - antessala da barbárie social amplificada [Entrevistado por Leslie Chaves]. Revista do Instituto Humanitas Unisinos UHU On-Line, 484, 44-49. Recuperado de http://www. ihuonline.unisinos.br/media/pdf/IHUOnlineEdicao484.pdf

Antunes, R., \& Praun, L. (2015). A sociedade dos adoecimentos no trabalho. Serviço Social \& Sociedade, 123, 407-427. doi: 10.1590/0101-6628.030 
Aquino, C. A. B. Sabóia, I. B., Melo, P. B., Carvalho, T. A., \& Ximenes, V. M. (2016). Terceirização e saúde do trabalhador: uma revisão da literatura nacional. Revista Psicologia Organizações e Trabalho, 16(2), 130-142. doi: 10.17652/rpot/2016.2.660

Bendassolli, P. F. (2011). Crítica às apropriações psicológicas do trabalho. Psicologia \& Sociedade, 23(1), 75-84. doi: 10.1590/ S0102-71822011000100009

Bendassolli, P. F., \& Guedes Gondim, S. M. (2014). Significados, sentidos e função psicológica do trabalho: discutindo essa tríade conceitual e seus desafios metodológicos. Avances en Psicología Latinoamericana, 32, 131-147. doi: http://dx.doi.org/10.12804/ apl32.1.2014.09

Biblioteca Virtual em Saúde - BVS. (2016). Descritores em Ciências da Saúde - DeCS [Base de dados]. Recuperado de http://decs.bvs.br/ cgi-bin/wxis1660.exe/decsserver/

Borges-Andrade, J. E., \& Zanelli, J. C. (2014). Psicologia e produção do conhecimento em organizações e trabalho. In J. C. Zanelli, J. E. Borges-Andrade, \& A. V. Bastos (Orgs.), Psicologia, Organizações e Trabalho no Brasil (pp. 583-608). Porto Alegre: Artmed.

Borges, L., Guimaraes, L., \& Silva, S. (2013). Diagnóstico e promoção da saúde psíquica no trabalho. In L. O. Borges \& L. Mourão (Orgs.), O trabalho e as organizações: atuações a partir da psicologia (pp. 581-618). Porto Alegre: Artmed.

Borsoi, I. C. F. (2007). Da relação entre trabalho e saúde à relação entre trabalho e saúde mental. Psicologia \& Sociedade, 19(n. esp.), 103111. doi: $10.1590 / S 0102-71822007000400014$

Centro de Referência Técnica em Psicologia e Políticas Públicas - CREPOP. (2008). Saúde do trabalhador no âmbito da saúde pública: referenciais para a atuação dos psicólogos. Brasília: Conselho Federal de Psicologia. Recuperado de http://crepop. pol.org.br/wp-content/uploads/2010/11/saude_do_trabalhador_ COMPLETO.pdf

Chaves, L. (2016). De volta aos tempos modernos no mundo do trabaIho?. Revista do Instituto Humanitas Unisinos UHU On-Line, 484, 12-13. Recuperado de www.ihuonline.unisino.br

Clot, Y. (2007). A função psicológica do trabalho (2ª ed.). Petrópolis: Vozes.

Costa, A. B., \& Zoltowski, A. P. (2014). Como escrever um artigo de revisão sistemática. In S. H. Koller, M. C. Couto, \& J. V. Hohendorff (Orgs.), Manual de produção científica. Porto Alegre: Penso.

Costa, D., Lacaz, F. A. C., Jackson Filho, J. M., \& Vilela, R. A. G. (2013). Saúde do trabalhador no SUS: desafios para uma política pública. Revista Brasileira de Saúde Ocupacional, 38(127), 11-21. doi: 10.1590/S0303-76572013000100003

Creswell, J.W. (2007). Projeto de pesquisa: métodos qualitativo, quantitativo e misto. Porto Alegre: Artmed.

Gondim, S. M. G., Borges-Andrade, J. E., \& Bastos, A. V. B. (2010). Psicologia do trabalho e das organizações: produção científica e desafios metodológicos. Psicologia em Pesquisa, 4(2), 84-99. Recuperado de http://pepsic.bvsalud.org/scielo.php?script=sci_arttext\&pid=S1982-12472010000200002\&Ing=pt\&tlng=pt.

Hohendorffi, J. V., DeSousa, D. A., Pereira, A. S., \& Koller, S. H. (2016) Nas "filas de espera": tempo entre submissão e aceitação de manuscritos em periódicos brasileiros de psicologia. Temas em Psicologia, 24(4), 1329-1341. doi: 10.9788/TP2016.4-08

Jacques, M. G. C. (2003). Abordagens teórico-metodológicas em saúde/doença mental \& trabalho. Psicologia \& Sociedade, 15(1), 97-116. doi: 10.1590/S0102-71822003000100006
Jardim, S. R., Ramos, A., \& Glina, D. M. (2010). Diagnóstico e nexo com o trabalho. In M. G. Jacques \& W. Codo (Orgs.), Saúde mental no trabalho: da teoria à prática (pp. 49-80). São Paulo: Roca.

Lacaz, F. A. C. (2007). O campo Saúde do Trabalhador: resgatando conhecimentos e práticas sobre as relações trabalho-saúde. Cadernos de Saúde Pública, 23(4), 757-766. doi: 10.1590/ S0102-311X2007000400003

Lacaz, F. A. C. (2016). Continuam a adoecer e morrer os trabalhadores: as relações, entraves e desafios para o campo Saúde do Trabalhador. Revista Brasileira de Saúde Ocupacional, 41. doi: 10.1590/2317-6369000120415

Leal, R. M. A. C., Bandeira, M. B., \& Azevedo, K. R. N. (2012). Avaliação da qualidade de um serviço de saúde mental na perspectiva do trabalhador: satisfação, sobrecarga e condições de trabalho dos profissionais. Psicologia: Teoria e Prática, 14(1), 15-25. Recuperado de: http://pepsic.bvsalud.org/scielo.php?script=sci_arttext\&pi$d=S 1516-36872012000100002 \&$ Ing $=p t \&$ tIng=pt.

Lei n. 13.429, de 31 de março de 2017 (2017, 31 de março). Altera dispositivos da Lei n. 6.019, de 3 de janeiro de 1974, que dispõe sobre o trabalho temporário nas empresas urbanas e dá outras providências; e dispõe sobre as relações de trabalho na empresa de prestação de serviços a terceiros. Diário Oficial da União, seção 1.

Lima, M. E. A. (2016, 16 de maio). Saúde mental e saúde do trabalhador: um diálogo indispensável. Recuperado de http://orientapsi.cfp. org.br/

Ministério da Educação, Coordenação de Aperfeiçoamento de Pessoal de Nível Superior. (2013). Critério Qualis por área 2013 (Psicologia) Recuperado de http://capes.gov.br/images/stories/download/avaliacaotrienal/Docs_de_area/Psicologia_doc_area_e_comiss\%C3\%A30_21out.pdf

Ministério da Educação, Coordenação de Aperfeiçoamento de Pessoal de Nível Superior. (2017). Cursos recomendados e reconhecidos / por região. Recuperado de https://sucupira.capes.gov.br/sucupira/ public/consultas/coleta/programa/quantitativos/quantitativoRegiao. jsf;jsessionid=IpC19tcuSCVdbQWNHKsjYjWE.sucupira-213

Moreira, D. S., Magnago, R. F., Sakae, T. M., \& Magajewski, F. R. L. (2009). Prevalência da síndrome de burnout em trabalhadores de enfermagem de um hospital de grande porte da Região Sul do Brasil. Cadernos de Saúde Pública, 25(7), 1559-1568. doi: 10.1590/ S0102-311X2009000700014

Navarro, V. L., \& Padilha, V. (2007). Dilemas do trabalho no capitalismo contemporâneo. Psicologia \& Sociedade, 19(n. esp), 14-20. doi: 10.1590/S0102-71822007000400004

Oliveira, J. R. S., Viganó, M. G., Lunardelli, M. C. F., Canêo, L. C., \& Goulart Júnior, E. (2010). Fadiga no trabalho: como o psicólogo pode atuar?. Psicologia em Estudo, 15(3), 633-638. doi: 10.1590/ S1413-73722010000300021

Osório da Silva, C. (2007). As ações de saúde do trabalhador como dispositivo de intervenção nas relações de trabalho. In E. M. Rosa, H. A. Novo, M. E. B. Barros, \& M. I. Moreira (Orgs.), Psicologia e saúde: desafios às políticas públicas no Brasil (pp. 75-90). Vitória: Edufes.

Pereira, M. G., \& Galvão, T. F. (2014). Extração, avaliação da qualidade e síntese dos dados para revisão sistemática. Epidemiologia e Serviços de Saúde, 23(3), 577-578. Recuperado de http://scielo.iec.pa.gov.br/scielo.php?script=sci_arttext\&pi$\mathrm{d}=$ S1679-49742014000300021\&lng=pt\&tIng=pt.

Periódicos Eletrônicos em Psicologia - PEPSIC [Base de dados]. (2017). Recuperado de http://pepsic.bvsalud.org/ 
propriações psicológicas do campo da saúde do trabalhador: Uma revisão sistemática

Portal Periódico Capes [Base de dados]. (2016). Recuperado de www. periodicoscapes.gov.br

Roque, H., Veloso, A., Silva, I., \& Costa, P. (2015). Estresse ocupacional e satisfação dos usuários com os cuidados de saúde primários em Portugal. Ciência \& Saúde Coletiva, 20(10), 3087-3097. doi: 10.1590/1413-812320152010.00832015

Rosado, I. V. M., Russo, G. H. A., \& Maia, E. M. C. (2015). Produzir saúde suscita adoecimento? As contradições do trabalho em hospitais públicos de urgência e emergência. Ciência \& Saúde Coletiva, 20(10), 3021-3032. doi: 10.1590/1413-812320152010.13202014

Sarriera, J. C., Moreira, M. C., Rocha, K. B., Bonato, T. N., Duso, R., \& Prikladnicki, S. (2003). Paradigmas em psicologia: compreensões acerca da saúde e dos estudos epidemiológicos. Psicologia \& Sociedade, 15(2), 88-100. doi: 10.1590/ S0102-71822003000200007
Sato, L., Lacaz, F. A. C., \& Bernardo, M. H. (2006). Psicologia e saúde do trabalhador: práticas e investigações na Saúde Pública de São Paulo. Estudos de Psicologia, 11(3), 281-288. doi: 10.1590/ S1413-294X2006000300005

Seligmann-Silva, E. (2011). Trabalho e desgaste mental: o direito de ser dono de si mesmo. São Paulo: Cortez.

Silva, S. C. P. S., Nunes, M. A. P., Santana, V. R., Reis, F. P., Machado Neto, J., \& Lima, S. O. (2015). A síndrome de burnout em profissionais da Rede de Atenção Primária à Saúde de Aracaju, Brasil. Ciência \& Saúde Coletiva, 20(10), 3011-3020. doi: 10.1590/1413-812320152010.19912014

Tonetto, A. M., Amazarray, M. R., Koller, S. H., \& Gomes, W. B. (2008). Psicologia organizacional e do trabalho no Brasil: desenvolvimento científico contemporâneo. Psicologia \& Sociedade, 20(2), 165-173. doi: 10.1590/S0102-71822008000200003

Cássio Adriano Braz de Aquino, Doutor em Psicologia Social pela Universidad Complutense de Madrid (UCM), Estágio Pós-doutoral no Departamento de Psicologia Social da Universidad Complutense de Madrid (UCM), é Professor Associado do Departamento de Psicologia da Universidade Federal do Ceará (UFC). Endereço para correspondência: Av. da Universidade, 2762 - Centro de Humanidades II, Benfica, Fortaleza, CE. CEP 60.020-180. E-mail: brazdeaquino@gmail.com

Mariana Aguiar Alcântara de Brito, Doutoranda do Programa de Pós-graduação em Psicologia da Universidade Federal do Ceará (UFC), é Professora da Universidade de Fortaleza (UNIFOR) e do Centro Universitário (UNI7). E-mail: marianapsiorg@gmail.com

Marselle Fernandes Fontenelle, Doutoranda do Programa de Pós-graduação em Psicologia da Universidade Federal do Ceará

(UFC), é Professora da Universidade de Fortaleza (UNIFOR)

E-mail: marsellefernandes@gmail.com

Maxmíria Holanda Batista, Doutoranda em Saúde Coletiva em Associação Ampla UFC-UECE-UNIFOR, é Professora da

Universidade de Fortaleza (UNIFOR).

E-mail: maxmiriaholanda@yahoo.com.br

Pamella Beserra de Melo, Mestre pelo Programa de Pós-graduação em Psicologia pela Universidade Federal do Ceará (UFC), é Psicóloga organizacional e do trabalho da Universidade Federal do Ceará (UFC). E-mail: pamellamelo_@hotmail.com

Samara Silva Silveira, Mestranda do Programa de Pós-graduação em Psicologia da Universidade Federal do Ceará (UFC), é Analista de Desenvolvimento de Pessoas da Companhia de Água e Esgoto do Estado do Ceará (CAGECE). E-mail: sss.samara@hotmail.com 